\title{
Focus point: cancer and HIV/AIDS dynamics-from optimality to modelling
}

\author{
Amar Debbouche $^{1, \mathrm{a}}\left(\mathbb{D}\right.$, Juan J. Nieto $^{2, \mathrm{~b}}{ }_{\mathbb{C}}$, Delfim F. M. Torres $^{3, \mathrm{c}}(\mathbb{D})$ \\ ${ }^{1}$ Department of Mathematics, Guelma University, Guelma, Algeria \\ 2 Institute of Mathematics, University of Santiago de Compostela, Santiago de Compostela, Spain \\ ${ }^{3}$ CIDMA, Department of Mathematics, University of Aveiro, Aveiro, Portugal
}

(C) The Author(s), under exclusive licence to Società Italiana di Fisica and Springer-Verlag GmbH Germany, part of Springer Nature 2021

Human cancer is a multistep process involving acquired genetic mutations, each of which imparts a particular type of growth advantage to the cell and ultimately leads to the development of a malignant phenotype. It is also a generic term for a group of diseases and figures as a leading cause of death globally; it lays a significant burden on healthcare systems and continues to be among the major health problems worldwide. The consequences of mutations in tumor cells include alterations in cell signaling pathways that result in uncontrolled cellular proliferation, insensitivity to growth inhibitory signals, resistance to apoptosis, development of cellular immortality, angiogenesis, tissue invasion and metastasis [1].

From the other side, acquired immunodeficiency syndrome (AIDS) is a chronic, potentially life-threatening condition caused by the human immunodeficiency virus (HIV). By damaging the immune system, HIV interferes with body's ability to fight infection and disease. HIV is a sexually transmitted infection (STI). It can also be spread by contact with infected blood or from mother to child during pregnancy, childbirth or breastfeeding. Without medication, it may take years before HIV weakens the immune system to the point that it has AIDS [2].

The current treatments still present substantial limitations: do not fully restore health and the offered medications are not curative. In fact, there is no cure or vaccine to the above deadly diseases, but medications can dramatically slow the progression of the disease. This serious problem motivated us to investigate this controversial subject.

This Focus Point, results after an open call for papers that considered substantially extended versions of papers presented at the conference ICMMAS'19 as well as external submissions. It covers the most promised new directions that were discussed and improved during the scientific meeting, together with interesting complementary novel articles. In particular, original results obtained from advanced theoretical, experimental and numerical simulations in all aspects related to cancer and HIV/AIDS dynamic systems, and explicitly meeting with the Aims and Scope of EPJP journal, were welcomed.

In light of: 2nd International Conference on Mathematical Modelling in Applied Sciences, August 20-24, 2019, Belgorod, Russia http://icmmas19.alpha-publishing.net.

\footnotetext{
a e-mail: amar_debbouche@yahoo.fr (corresponding author)

b e-mail: juanjose.nieto.roig@usc.es

c e-mail: delfim@ua.pt
} 
In the first paper [3], the authors investigated a randomized discrete logistic equation to describe the dynamics of breast tumor volume. They appropriately assigned reliable distributions to model inputs and sample data and also designed a computational procedure to determine parameters, and then they applied to model the breast tumor volume using real data.

In second contribution [4], a mathematical model of breast cancer involving a system of differential equations with piecewise constant arguments is considered to analyze the tumor growth and chemotherapeutic treatment. They established the competition among both normal and tumor cells. Sensitivity property is considered for the stability and oscillation behaviors. They also incorporated the Allee effect at time to analyze the breast cancer population for the extinction case.

In the third paper [5], the dynamical behavior of a mathematical model of HIV influenced by stochastic perturbations is studied. It explores the disease-free equilibrium's stochastic global exponential stability. It also provides stability areas and numerical simulations to confirm the analytical results by using the fundamental Euler-Maruyama (EM) algorithm.

In the followed investigation [6], authors incorporate the Beddington-DeAngelis incidence rate to a continuous-time HIV infection model with cure rate and a full logistic proliferation rate. Equilibria and their local stability analysis are treated. Moreover, an optimal control strategy is applied to the discrete-time model in order to reduce the number of infected cells as well as the number of free HIV particles. Numerical simulations are also performed to verify the theoretical analysis obtained.

The next research [7] presents a model of the tumor and normal cell growth under the influence of a carcinogenic agent, an immunomodulator (IM) and variable influx of immune cells including relevant interactions. The tumor growth is facilitated by carcinogens, and it observes to have considerable advantage over the constant influx model. The evolutions of normal and tumor cells are computed and numerically simulated.

AlShamrani et al. [8] investigated a within-host HIV dynamics under the effect of cytotoxic T lymphocytes immune response. They incorporated two modes of transmission: virus to cell (VTC) and cell to cell (CTC), and integrated three types of distributed time delays. Wellposedness, equilibria stability and numerical simulations are detailed.

In [9], Babaei, Jafari and Liya examined the interaction between drug addiction and the contagion of HIV/AIDS in Iranian prisons. Stability and treatment effects of drug users on the spread of HIV/AIDS are pointed out. Rehabilitation impact on the control of HIV/AIDS spread is well investigated, and the reproduction numbers are compared in cases where there is no cure or some treatment methods are available.

The research work [10] considered a system of fractional differential equations with delays and presented a computational method based on hybrid functions of general block-pulse and Legendre polynomials. Error estimate analysis was also studied. Moreover, two models, arising in engineering and applied science, including the Chen and human immunodeficiency viruses systems, were applied.

Moreover, in [11] the authors treat the second most common cancer in females and a major cause of death in the world nowadays, which is cervical cancer. Detections, diagnosis and screening were discussed. It gives a framework for understanding how early diagnosis increases the chances for cancer patients to be cancer free by providing a proper treatment. Local stability of the equilibrium points of systems in the presence and absence of diagnosis was analyzed and compared. It is also enhanced the role of diagnosis by using optimal control strategies to prevent the cervical cancer.

Further, the paper [12] considered an HIV/AIDS epidemic model including five compartments under fractal-fractional-order derivative. Existence, Ulam-Hyers and generalized 
stability conditions via nonlinear functional analysis are established. Moreover, a fractional Adams-Bashforth method is developed for numerical simulation and applied on some existing real data of disease spread in South Africa. It is shown that the infected individuals, who have changed their sexual habits sufficiently after starting the treatment and changing their sexual behavior, increased gradually.

Furthermore, the research team [13] describes the dynamics of HIV-AIDS infection by investigating the celebrated mathematical SICA model via a fractional order system. Stability and instability of equilibrium points are determined in terms of the basic reproduction number. Additionally, a fractional optimal control system is formulated and suitable strategies for minimizing the spread of the disease into the population are determined.

In the last contribution, [14], the authors prove sufficient conditions for the stability of a stationary solution to a system of nonlinear partial differential equations of the diffusion model describing the growth of malignant tumors. They also numerically simulate stable and unstable scenarios involving the interaction between tumor and immune cells.

We take this opportunity to thank all the contributors of FP: Cancer \& HIV/AIDS Dynamics: From Optimality to Modelling, more precisely, to all authors for confirming their interests to the subject, by submitting their works for possible publication, and also to all referees for helping our editorial task. A special thanks and respect goes to Prof. Dr. Paolo Biscari, Editor in Chief of EPJP, and also to Springer for accepting our proposal and giving us a green light to form this issue.

\section{References}

1. WHO-World Health Organization, Cancer: Fact Sheets. Web page available at: https://www.who.int/ news-room/fact-sheets/detail/cancer (2020)

2. C. Scott, M.D. Litin, in Mayo Clinic Family Health Book (Time Incorporated Home Entertainment, 2009)

3. C.B. Simón, J.C. Cortés, D.M. Rodríguez, R.J. Villanueva, Eur. Phys. J. Plus 135, 826 (2020)

4. F.B. Yousef, A. Yousef, T. Abdeljawad, A. Kalinli, Eur. Phys. J. Plus 135, 952 (2020)

5. H. El-Metwall, M.A. Sohaly, I.M. Elbaz, Eur. Phys. J. Plus 135, 840 (2020)

6. S.M. Salman, Eur. Phys. J. Plus 135, 808 (2020)

7. R. Kiran, S. Tyagi, S. Abbas, M. Roy, A. Taraphder, Eur. Phys. J. Plus 135, 735 (2020)

8. N.H. AlShamrani, A.M. Elaiw, H. Dutta, Eur. Phys. J. Plus 135, 593 (2020)

9. A. Babaei, H. Jafari, A. Liya, Eur. Phys. J. Plus 135, 395 (2020)

10. K. Nouri, M. Nazari, L. Torkzadeh, Eur. Phys. J. Plus 135, 341 (2020)

11. S. Chakraborty, A. Debbouche, V. Antonov, Eur. Phys. J. Plus 135, 780 (2020)

12. Z. Ali, F. Rabiei, K. Shah, T. Khodadadi, Eur. Phys. J. Plus 136, 36 (2021)

13. A. Boukhouima, E. Lotfi, M. Mahrouf, S. Rosa, D.F.M. Torres, N. Yousfi, Eur. Phys. J. Plus 136, 103 (2021)

14. A. Debbouche, M.V. Polovinkina, I.P. Polovinkin, C.A. Valentim Jr., S.A. David, Eur. Phys. J. Plus 136, $131(2021)$ 